\title{
EL AULA PROFESIONAL DE EMPRENDIMIENTO DE LA UNIVERSIDAD COMPLUTENSE DE MADRID: APRENDER Y EMPRENDER
}

\author{
THE PROFESSIONAL ENTREPRENEURSHIP SPACE \\ OF THE COMPLUTENSE UNIVERSITY OF MADRID: \\ LEARNING AND ENTREPRENEURSHIP
}

\section{Carolina Fernández-Salinero Miguel ${ }^{a}$, Beatriz De La Riva-Picatoste ${ }^{a}$ y Ángela Martín-Gutiérrez ${ }^{b}$}

Fechas de recepción y aceptación: 5 de junio de 2021 y 7 de julio de 2021

DOI: https://doi.org/10.46583/edetania_2021.60.899

Resumen: Conseguir el desarrollo y promoción de la educación emprendedora es uno de los objetivos políticos clave de la Unión Europea (UE) y de sus Estados miembros desde los años noventa del siglo pasado. Para dar respuesta a este objetivo surge el proyecto Aulas Profesionales de Emprendimiento (APES) de la Comunidad de Madrid, que inicialmente se establece en formación profesional reglada, pero que poco a poco se está empezando a poner en marcha también en la Universidad, constituyéndose como una estrategia para impulsar la competencia emprendedora, imprescindible en el nuevo marco socioeconómico hacia el que se dirige nuestra economía. Concretamente, en la Facultad de Educación-Centro de Formación del Profesorado (UCM) se ha puesto en marcha una APE. Se trata de una actuación de carácter eminentemente práctica, encaminada a potenciar el emprendimiento y el autoempleo entre el estudiantado y concebida como una preincubadora que pretende integrarse en el ecosistema emprendedor de la UCM, concretamente, en la red de preincubadoras de la Universidad. El alumnado se acerca a ella para adquirir las competencias transversales necesarias para la ciudadanía activa, la empleabilidad y el emprendimiento, así como para transformar propuestas creativas en acciones emprendedoras. A partir de ese momento, el estudiantado encuentra los

\footnotetext{
${ }^{a}$ Facultad de Educación. Centro de Formación del Profesorado. Universidad Complutense de Madrid (UCM).

${ }^{*}$ Correspondencia: Universidad Complutense de Madrid. Facultad de Educación. Centro de Formación del Profesorado. Calle Rector Royo-Villanova, s/n. 28040 Madrid. España.

E-mail: cfernand@ucm.es

${ }^{\mathrm{b}}$ Universidad Internacional de La Rioja.
} 
recursos necesarios para poder iniciar su andadura por la vía del emprendimiento: un mentor encargado del asesoramiento, una formación instrumental adecuada y un espacio físico dotado de equipos informáticos (la hiperaula), o un espacio virtual, en nuestro caso dentro de la página web de la facultad.

Palabras clave: Aulas Profesionales de Emprendimiento, ecosistema emprendedor, educación superior, emprendimiento, preincubadora.

Abstract: Achieving the development and promotion of entrepreneurial education is one of the key political goals of the European Union (EU) and its Member States since the nineties of the last century. In response to this goal, the "Aulas Profesionales de Emprendimiento" (APES) project of the Community of Madrid was created, which was initially established in Regulated Vocational Training, but is gradually beginning to be implemented in universities as well, constituting a strategy to promote entrepreneurial skills, essential for the new socioeconomic framework towards which our economy is heading. Specifically, an APE has been set up at the Faculty of Education-Teaching Training Center (UCM). It is an eminently practical action, aimed at promoting entrepreneurship and self-employment among students and conceived as a pre-incubator that aims to integrate into the entrepreneurial ecosystem of the UCM, specifically, in the network of pre-incubators of the university. Students approach it to acquire the transversal skills necessary for active citizenship, employability and entrepreneurship, and to transform creative proposals into entrepreneurial actions too. From that moment on, students find the necessary resources to start their journey through entrepreneurship: a mentor in charge of counseling, an adequate instrumental training and a physical space equipped with computer equipment (the hyper-classroom) or a virtual space, in our case within the website of the Faculty.

Keywords: Professional Entrepreneurship Space, entrepreneurial ecosystem, higher education, entrepreneurship, pre-incubator.

\section{INTRODUCCIÓN}

En el contexto actual de alta tasa de desempleo juvenil, crisis económica y rapidez de cambios aparejados a nuestra compleja economía y sociedad del conocimiento, las competencias transversales y, particularmente, el emprendimiento son esenciales para conseguir que la juventud llegue a convertirse en una ciudadanía activa, creativa y emprendedora. La educación emprendedora podemos definirla, desde esta perspectiva, como la conexión con un proyecto de educación humanizadora, donde se educaría al estudiantado en la iniciativa, autonomía, responsabilidad y madurez personal, para que valore, 
piense, invente, cree e imagine nuevas fórmulas y proyectos personales para afrontar los amplios desafíos que se nos plantean, incluidos los relacionados con el mundo del trabajo (Mayor-Zaragoza, 2009). Es un enfoque educativo que posibilita el crecimiento del potencial emprendedor del alumnado y que pretende contribuir en un futuro al crecimiento integral, principalmente de las dimensiones intelectual, societaria y moral de la persona, no limitándose al crecimiento socioeconómico y laboral (Azqueta y Naval, 2019; Suárez-Ortega, Sánchez-García y Soto-González, 2020).

Este enfoque es el que subyace a la aportación práctica del emprendimiento universitario que analizamos en este trabajo, partiendo de la visión supranacional de la Unión Europea, de la actuación emprendedora promovida por la universidad española y de las propuestas concretas de determinadas facultades como la que se presenta aquí: la Facultad de Educación-Centro de Formación del Profesorado (CFP) de la Universidad Complutense de Madrid (UCM). No se trata de analizar los programas concretos de emprendimiento que se están llevando a cabo en la Universidad española, sino más bien de describir y dar a conocer los servicios y propuestas de emprendimiento que se están implementando de manera transversal en los estudios superiores de nuestro país, parándonos específicamente en uno de ellos, cuyo interés radica en el ámbito en el que se desarrolla (la Facultad de Educación-CFP), la forma en que se plantea (Aula Profesional de Emprendimiento) y el tipo de emprendimiento que propone (iniciación al emprendimiento y creación de negocio).

Tres son los objetivos que persigue este trabajo: identificar la situación actual del emprendimiento universitario, auspiciado por las políticas y planes de la Unión Europea y asumido por las universidades españolas; visibilizar el interés mostrado por la universidad española en desarrollar propuestas transversales de apoyo al emprendimiento; y analizar una propuesta concreta de emprendimiento universitario en la Facultad de Educación-CFP(UCM).

La metodología que nos sirve de base para conseguir dichos objetivos es, si seguimos a Bisquerra (2009), fundamentalmente de carácter descriptivointerpretativo, se encuadra en un enfoque cualitativo y se apoya en la técnica de análisis documental (útil para desarrollar las dos primeras partes del artículo) y de estudio de caso (adecuada para analizar el Aula Profesional de Emprendimiento de la Facultad de Educación-CFP de la UCM). 


\section{EMPRENDimiENTO EN El CONTEXTO EUROPEO, NACiONAL y LOCAL}

El Consejo Europeo de Lisboa del año 2000 se puede considerar como el momento en el cual se empieza a vislumbrar el interés por impulsar la formación emprendedora en Europa, facilitando la puesta en marcha de las ideas que Delors ya planteó en 1993 en su Libro Blanco. Hitos posteriores reivindican la continuidad de este interés, destacándose a continuación los siguientes (Redie, 2015; Azqueta, 2021):

- Carta Europea de la Pequeña Empresa (2000).

- Foro "Formar para emprender" (2000).

- Procedimiento BEST sobre educación y formación en el espíritu empresarial (2002).

- Libro Verde sobre el Espíritu Empresarial en Europa (2003).

- Programa europeo a favor del espíritu empresarial (2004).

- Comunicación "Fomentar la mentalidad empresarial mediante la educación y la formación" (2006).

- Conferencia y agenda de Oslo para la educación en iniciativa empresarial en Europa: el fomento del espíritu empresarial a través de la educación y la formación (2006).

- Programa marco para la innovación y la competitividad (2006).

- Recomendación 2006/962/CE del Parlamento Europeo y del Consejo y su actualización a través de la Recomendación 2018/C189/01 del Consejo, sobre las competencias clave para el aprendizaje permanente, entre ellas la competencia emprendedora.

- Emprendimiento en educación superior, especialmente en carreras fuera del ámbito económico-empresarial (2008).

- The European Entrepreneurial Region (2009).

- Grupo de expertos "hacia una mayor cooperación y coherencia en la educación emprendedora" (2010).

- Plan de acción Emprendimiento 2020: relanzar el espíritu emprendedor en Europa (2012).

- Guía Cómo crear mentalidades y capacidades emprendedoras en la Unión Europea (2013).

- Educación para el emprendimiento. Guía del educador (2014). 
- Conclusiones del Consejo sobre el emprendimiento en la educación y formación (diciembre de 2014).

- Educación Emprendedora: El camino hacia el éxito. Compendio de evidencias de educación emprendedora en Europa (2015).

- Informe sobre Educación y emprendimiento en Europa (2016).

Por tanto, conseguir el desarrollo y la promoción de la educación emprendedora es uno de los objetivos políticos clave de la Unión Europea (UE) y de sus Estados miembros. A este respecto, Bourgeois (2011) destaca la relevancia del espíritu empresarial no solo para formar la mentalidad de la juventud, sino también para proporcionar los conocimientos, las habilidades y las actitudes que son fundamentales para desarrollar una cultura empresarial en Europa que dé respuesta al elevado desempleo juvenil, las crisis económicas y los rápidos cambios relacionados con la complejidad social y económica en la que nos encontramos inmersos. El "programa Erasmus para jóvenes empresarios" y el "programa Yep" son dos ejemplos de ello.

En el contexto español, específicamente en el ámbito universitario que es el que nos ocupa, encontramos experiencias de buenas prácticas dirigidas al establecimiento de centros de asesoramiento e incubación de ideas de negocio. A este respecto, la tabla 1 presenta las universidades españolas que tienen servicios o infraestructuras dedicadas al emprendimiento de su alumnado.

TABLA 1

Infraestructuras emprendedoras en Educación Superior en España

\begin{tabular}{|l|l|}
\hline \multicolumn{1}{|c|}{$\begin{array}{c}\text { Comunidad } \\
\text { autónoma }\end{array}$} & \multicolumn{1}{c|}{ Universidades con infraestructura emprendedora } \\
\hline Andalucía & $\begin{array}{l}\text { Universidad de Almería, Cádiz, Córdoba, Granada, Huelva, Internacional de An- } \\
\text { dalucía, Jaén, Loyola Andalucía, Málaga, Pablo de Olavide Sevilla y Universidad } \\
\text { de Sevilla. }\end{array}$ \\
\hline Aragón & Universidad San Jorge Grupo Sanvalero y Universidad de Zaragoza. \\
\hline Asturias & Universidad de Oviedo. \\
\hline Islas Baleares & Universitat de les Illes Balears. \\
\hline Islas Canarias & Universidad de La Laguna y Universidad de las Palmas de Gran Canaria. \\
\hline Cantabria & Universidad de Cantabria. \\
\hline
\end{tabular}




\begin{tabular}{|c|c|}
\hline $\begin{array}{l}\text { Comunidad } \\
\text { autónoma }\end{array}$ & Universidades con infraestructura emprendedora \\
\hline $\begin{array}{l}\text { Castilla-La } \\
\text { Mancha }\end{array}$ & Universidad de Castilla-La Mancha. \\
\hline Castilla y León & $\begin{array}{l}\text { Universidad Católica de Ávila, Burgos, Europea Miguel de Cervantes, León, Sala- } \\
\text { manca, Pontificia de Salamanca y Valladolid. }\end{array}$ \\
\hline Cataluña & $\begin{array}{l}\text { Universitat Abat Oliba CEU, Central de Barcelona, Autònoma de Barcelona, Oberta } \\
\text { de Catalunya, Politècnica de Catalunya-Barcelonatech, Girona, Internacional de Ca- } \\
\text { talunya, Lleida, Pompeu Fabra Barcelona, Ramon Llul, Rovira i Virgili y Universitat } \\
\text { de Vic-Universitat Central de Catalunya. }\end{array}$ \\
\hline Extremadura & Universidad de Extremadura. \\
\hline Galicia & Universidade da Coruña, Santiago de Compostela y Vigo. \\
\hline La Rioja & Universidad de la Rioja e Internacional de la Rioja. \\
\hline Madrid & $\begin{array}{l}\text { Universidad de Alcalá de Henares, Alfonso X El Sabio, Camilo José Cela, Carlos } \\
\text { III de Madrid, Complutense de Madrid, Europea de Madrid, Francisco de Vitoria de } \\
\text { Madrid, Reinventing Higher Education, Internacional Menéndez Pelayo, Autóno- } \\
\text { ma de Madrid, A Distancia de Madrid, Politécnica, Nebrija, Comillas Universidad } \\
\text { Pontificia, Rey Juan Carlos y CEU Universidad San Pablo. }\end{array}$ \\
\hline Murcia & Universidad Politécnica de Cartagena, Murcia y Católica de Murcia. \\
\hline Navarra & Universidad de Navarra y Pública de Navarra. \\
\hline País Vasco & Universidad de Deusto, Mondragón Unibertsitatea y Universidad del País Vasco. \\
\hline $\begin{array}{l}\text { Comunidad } \\
\text { Valenciana }\end{array}$ & $\begin{array}{l}\text { Universitat d'Alicant, CEU Universidad Cardenal Herrera, Universitat Jaume I, } \\
\text { Universitas Miguel Hernández, Universidad Católica de Valencia San Vicente Mártir, } \\
\text { Universitat de València y Universitat Politècnica de València. }\end{array}$ \\
\hline
\end{tabular}

Fuente: elaboración propia

La tabla 1 nos aporta información sobre el ecosistema de emprendimiento que se está implementando en la Universidad española, pública y privada. Un ecosistema que tiene como objetivos: potenciar la cultura emprendedora, promover la empleabilidad, incrementar las relaciones de la Universidad con el entorno socioeconómico y desarrollar la innovación. Para conseguir estos objetivos se ponen en marcha recursos informativos, formativos, tecnológicos y financieros, y se crean espacios de coworking y preincubación, así como viveros de empresas. Todo ello con un mayor o menor desarrollo según las 
características y el tamaño de las universidades, un carácter más empresarial o de promoción de competencias emprendedoras en el alumnado, un enfoque más o menos social del emprendimiento y una organización más general o por facultades. Pero en todos los casos con un marcado interés por el asesoramiento y apoyo al estudiantado universitario en pos de conseguir su inserción efectiva en el mercado laboral.

En este contexto, más concretamente en la Comunidad Autónoma de Madrid, destaca el "proyecto Aulas Profesionales de Emprendimiento (APE)". Este proyecto surge inicialmente enfocado a la Formación Profesional (FP), para impulsar las competencias emprendedoras, imprescindibles en el nuevo marco socioeconómico, pero poco a poco comienza a transferirse al contexto universitario, de ahí su interés. Las APE persiguen actuaciones de carácter eminentemente práctico, encaminadas a potenciar el emprendimiento y el autoempleo entre el estudiantado. En este sentido, funcionan como espacios educativos, físicos y temporales, en los que el alumnado puede adquirir competencias y desarrollar sus ideas de negocio poniendo en práctica la formación recibida en clase y sometiendo a la experiencia los conocimientos adquiridos.

La Comunidad de Madrid puso en marcha la propuesta, que fue aprobada en la Asamblea el 22 de septiembre de 2017, promoviendo así la creación de espacios de emprendimiento en los centros de FP bajo la coordinación del profesorado de Formación y Orientación Laboral (FOL). De esta manera, en el curso 2017-2018 se inició de forma experimental en cinco centros públicos de FP. Se trataba de un proyecto voluntario, por lo que cada centro podía decidir si implantaba o no este tipo de aula. No obstante, a partir del curso 2018-2019 fueron sumándose más centros a esta iniciativa gracias al Decreto 63/2019, de 16 de julio, del Consejo de Gobierno, por el que se regula la ordenación y organización de la formación profesional en la Comunidad de Madrid. En un comunicado de la comunidad se hace explícito que, tras la promulgación del decreto, se contó con 27 APE que disponían de los medios humanos y materiales para llevar a cabo los proyectos del alumnado: un mentor para el asesoramiento y un espacio físico dotado de equipos informáticos.

Tal y como indica el Decreto 63/2019 (desarrollado actualmente a través de la Orden 1378/2021, de 19 de mayo), las APE ofrecen al alumnado una doble línea de aprendizaje y oportunidades: 
- Facilitar los instrumentos necesarios para la constitución de microempresas a partir de la formación recibida en el aula, proporcionando un cauce de inserción en el mercado laboral. De este modo, se promueve la actividad empresarial como alternativa válida y atractiva al trabajo por cuenta ajena, lanzando al mercado empresas viables capaces de ofrecer productos y servicios competitivos en los distintos sectores económicos.

- Transmitir al alumnado los aprendizajes esenciales, que se adquirirán fundamentalmente a través de las competencias personales y profesionales, imprescindibles para desarrollar el perfil emprendedor, potenciando también sus opciones de profesionalización futuras.

Como destinatario de las APE se encuentra el estudiantado de los centros educativos que esté cursando FP (Básica, Media y Superior) y el alumnado egresado de FP en los tres años siguientes a la finalización de sus estudios.

Este proyecto ha empezado poco a poco a ponerse en marcha también en la Universidad, fundamentalmente en el ámbito privado (un ejemplo sería el Aula de Emprendedores de la Universidad Pontificia de Comillas), constituyéndose como una estrategia para impulsar la competencia emprendedora en el espacio europeo de educación superior, imprescindible en el nuevo marco socioeconómico hacia el que se dirige nuestra economía. En esta línea surge el Aula Profesional de Emprendimiento de la Facultad de Educación-Centro de Formación del Profesorado (CFP) de la UCM (APE-Educación UCM), concebida como una preincubadora que pretende integrarse en el ecosistema emprendedor de la UCM. Es una experiencia innovadora a la que nos vamos a referir a continuación.

\section{El ecosistema emprendedor de la Universidad Complutense DE MADRID}

La trascendencia de orientar la educación universitaria a la promoción del emprendimiento estriba en el reconocimiento de la importancia de este tipo especial de actividad para el desarrollo integral de la sociedad y de la economía (Vázquez, 2017). Morales, Mira y Arias (2010) plantean, a este respecto, 
que una de las funciones básicas de la Universidad debe ser vincularse a la empresa, hecho propio de los países en desarrollo y de las universidades de Europa occidental y de Estados Unidos. Esta idea ha influido en que las universidades se hayan interesado en fomentar la cultura del emprendimiento por tratarse de una estrategia para transferir conocimiento al entorno (Sanabria, Morales y Ortiz, 2015).

La UCM, como una de las universidades de Europa occidental, tiene encomendada, entre sus funciones, la preparación para el ejercicio de actividades profesionales, así como la difusión, valorización y transferencia del conocimiento al servicio de la cultura, de la calidad de vida y del desarrollo económico, y resulta necesario en este contexto un espacio destinado a acciones de emprendimiento (Decreto Rectoral 1/2007). Dicho espacio se conforma en 2007 como Compluemprende, que nace como un lugar para vincular a todos los agentes de la UCM que pudiesen estar relacionados con el emprendimiento y la cultura emprendedora. Las actividades de esta entidad, tal y como se desprende de sus memorias anuales publicadas en los últimos años (2017 y 2019), quedan resumidas en la siguiente tabla:

TABLA 2

Actividades y acciones desarrolladas por Compluemprende

\begin{tabular}{|l|l|l|}
\hline \multicolumn{1}{|c|}{ Acciones de formación } & \multicolumn{1}{|c|}{ Acciones de información } & \multicolumn{1}{c|}{ Otras acciones } \\
\hline $\begin{array}{l}\text { Tutorización de proyectos } \\
\text { de emprendimiento. }\end{array}$ & $\begin{array}{l}\text { Apoyo al proceso de creación de } \\
\text { empresas. }\end{array}$ & $\begin{array}{l}\text { Firma de convenios de colabora- } \\
\text { ción con diferentes entidades. }\end{array}$ \\
\hline $\begin{array}{l}\text { Coordinación de programas } \\
\text { de emprendimiento. }\end{array}$ & $\begin{array}{l}\text { Ofrecimiento de información a } \\
\text { través de los diferentes puntos de } \\
\text { asesoramiento. }\end{array}$ & $\begin{array}{l}\text { Entrega de premios para empren- } \\
\text { dedores y participación en premios } \\
\text { nacionales e internacionales. }\end{array}$ \\
\hline $\begin{array}{l}\text { Diploma de emprendimien- } \\
\text { to cursando asignaturas } \\
\text { trasversales. }\end{array}$ & $\begin{array}{l}\text { Asesoramiento a investigadores } \\
\text { para la creación de empresas con } \\
\text { base tecnológica. }\end{array}$ & $\begin{array}{l}\text { Fomento de la vocación empren- } \\
\text { dedora. }\end{array}$ \\
\hline $\begin{array}{l}\text { Talleres formativos. } \\
\text { Orientación a proyectos de ne- } \\
\text { gocio. }\end{array}$ & $\begin{array}{l}\text { Establecimiento de un entorno en el } \\
\text { que adquirir y mejorar las capaci- } \\
\text { dades empresariales. }\end{array}$ \\
\hline $\begin{array}{l}\text { Visitas a ecosistemas em- } \\
\text { prendedores. }\end{array}$ & $\begin{array}{l}\text { Difusión enfocada a motivar el } \\
\text { emprendimiento. }\end{array}$ & $\begin{array}{l}\text { Supervisión de programas de em- } \\
\text { prendimiento. }\end{array}$ \\
\hline
\end{tabular}

Fuente: elaboración propia a partir de las memorias publicadas por Compluemprende en 2017 y 2019 
Además, Compluemprende, en su afán por apoyar al estudiante de la Universidad Complutense y en busca de la mejora de su tarea difusora, realiza acciones directamente vinculadas a las distintas facultades de la Universidad. Entre estas acciones cabe destacar:

a) Las actividades formativas ligadas directamente a las facultades, destinadas al estudiantado y también al profesorado, como los talleres de salidas profesionales y los seminarios sobre emprendimiento.

b) La red de preincubadoras intercentros (RPIC-UCM). Desde Compluemprende se apoya a las facultades con el objetivo de crear una red de preincubadoras, que actualmente son tres, como se puede comprobar en la siguiente tabla.

TABLA 3

Red de preincubadoras intercentros de la UCM

\begin{tabular}{|l|c|l|}
\hline \multicolumn{1}{|c|}{ Preincubadoras } & Año de inicio & \multicolumn{1}{c|}{ Objetivos } \\
\hline $\begin{array}{l}\text { Preincubadora BIO } \\
\text { de la Facultad de } \\
\text { Ciencias Biológicas }\end{array}$ & 2014 & $\begin{array}{l}\text { - Promover el emprendimiento y potenciar iniciativas em- } \\
\text { prendedoras. }\end{array}$ \\
\hline $\begin{array}{l}\text { Asesorar a emprendedores universitarios para que definan } \\
\text { su producto o servicio, resuelvan sus problemas y cubran } \\
\text { sus necesidades. }\end{array}$ \\
\hline $\begin{array}{l}\text { Preincubadora } \\
\text { Facultad de Ciencias } \\
\text { Económicas y Em- } \\
\text { presariales }\end{array}$ & 2016 & $\begin{array}{l}\text { - Acercar al estudiantado universitario al mundo empresarial. } \\
\text { - Facilitar información al alumnado para la iniciación de } \\
\text { proyectos. }\end{array}$ \\
\hline $\begin{array}{l}\text { Par a emprendedores universitarios la oportunidad de } \\
\text { Facultad de Comer- } \\
\text { formarse. }\end{array}$
\end{tabular}

Fuente: elaboración propia

c) Red de embajadores: profesorado y personal investigador de la Universidad que difunden el emprendimiento en sus distintas facultades.

Actualmente, Compluemprende se inscribe dentro de una estructura mayor que es el Vicerrectorado de Empleabilidad y Emprendimiento, entre cuyas funciones están todas las cuestiones relativas a empleabilidad, emprendimiento 
y prácticas externas (Decreto Rectoral 1/2021). Este Vicerrectorado pretende responder además a lo establecido en el Plan de Recuperación, Transformación y Resiliencia presentado por el Gobierno de España en abril de 2021, el cual ha supuesto la puesta en marcha de medidas urgentes para la recuperación económica tras la crisis provocada por la COVID-19, entre las que podemos destacar las siguientes:

- El impulso del emprendimiento orientado a favorecer las oportunidades profesionales de la juventud.

- El Programa de Orientación, Avance y Emprendimiento Educativo para apoyar a los centros de especial complejidad educativa.

- La modernización del sistema universitario y la mejora de las infraestructuras digitales y equipamientos universitarios fomentando la excelencia, la transición tecnológica y las sinergias entre la investigación, la formación universitaria y el emprendimiento.

Es en este ecosistema emprendedor en el que el Aula Profesional de Emprendimiento de la Facultad de Educación-CFP (UCM) inicia su andadura para establecerse como preincubadora en el sector de la educación. A ella vamos a referirnos específicamente a continuación.

\section{APE-EDUCACIÓN UCM: UNA PROPUESTA DE EMPRENDIMIENTO UNIVERSITARIO}

El Aula Profesional de Emprendimiento de la Facultad de Educación-CFP de la Universidad Complutense de Madrid (APE-Educación UCM) se concibe como una preincubadora que pretende integrarse en el ecosistema emprendedor de la UCM y que se pone en funcionamiento el curso 2020/2021, de manera virtual por exigencias sanitarias, gracias a la concesión de un Proyecto Innova Docencia, promovido y financiado por el Vicerrectorado de Calidad de la UCM. Este tipo de proyectos están destinados a la mejora de la calidad docente vinculada a la innovación, de manera que consigue que toda la comunidad universitaria se implique en el progreso de la institución y en el servicio que ofrece. 
Este proyecto surge de la preocupación de dos docentes del Departamento de Estudios Educativos de la Facultad de Educación-CFP(UCM), embajadoras de emprendimiento UCM, por promover contenidos de emprendimiento en los grados universitarios de educación, por facilitar un espacio para su desarrollo dentro de la propia Facultad (físicamente en la hiperaula y virtualmente en la página web del centro) que permita el intercambio de ideas y experiencias, por favorecer las posibilidades de inserción laboral del alumnado de la Facultad de Educación-CFP y por llegar a formar parte de la Red de Preincubadoras de la UCM. El APE-Educación UCM es además la única aula profesional de emprendimiento universitario vinculada a una facultad de Educación en la Comunidad Autónoma de Madrid.

El APE-Educación UCM se crea, por tanto, con el objetivo de promover el autoempleo del alumnado universitario como alternativa al trabajo por cuenta ajena y, además, favorecer la adquisición de los conocimientos básicos acerca de lo que es el emprendimiento, las destrezas sociales necesarias para poder utilizar dichos conocimientos, así como las actitudes y aptitudes imprescindibles para considerarlo como una alternativa profesional. El estudiantado se acerca a ella para adquirir las competencias transversales necesarias para la ciudadanía activa, la empleabilidad y el emprendimiento, así como para transformar propuestas creativas en acciones emprendedoras, al acudir a esta con una idea de negocio sobre la que les interesa trabajar con la intención de llegar a convertirla en una empresa. El alumnado encuentra en el APE-Educación UCM los recursos necesarios para poder iniciar su andadura por la vía del emprendimiento: un mentor encargado del asesoramiento, una formación instrumental adecuada $\mathrm{y}$, este curso, un espacio virtual como entorno de desarrollo ${ }^{1}$.

El equipo del proyecto reúne diferentes tipos de perfiles. Por un lado, está el personal docente e investigador, compuesto por dos docentes con dedicación a tiempo completo y una docente con dedicación a tiempo parcial. Profesorado que, además, pertenece a la misma área de conocimiento (Teoría e Historia de la Educación) y está vinculado al mismo departamento (Estudios Educativos). Por otro lado, en el equipo hay dos profesionales del mundo de la empresa, uno de ellos también vinculado a la Universidad como profesor asociado del

\footnotetext{
${ }^{1}$ Se ha creado, a este respecto, la página web del APE-Educación UCM, cuyo enlace es el siguiente: https://www.ucm.es/emedu/.
} 
Departamento de Marketing del Grado en ADE, en la Universidad Pública de Navarra, y el otro trabajando como business angel. Finalmente, está el alumnado participante en el proyecto, consistente en una estudiante del grado en Pedagogía y un alumni (antiguo alumno de la UCM) del mismo grado, así como una alumna de doctorado, a punto de finalizar su tesis, que además tiene un contrato predoctoral de formación de profesorado universitario (FPU).

\subsection{Objetivos}

Los objetivos planteados en este proyecto se dividen en generales y específicos:

Los generales son:

- Promover un trabajo interdisciplinar.

- Sensibilizar al profesorado y al alumnado de la Facultad de EducaciónCFP (UCM) acerca de la importancia del emprendimiento, tanto interno (desarrollo de la competencia emprendedora) como externo (creación de un negocio).

Y los específicos:

- Crear un aula profesional de emprendimiento (APE-Educación UCM) en la Facultad de Educación-CFP (UCM), físicamente en las instalaciones de la hiperaula y virtualmente en la página web de la Facultad, que llegue a formar parte de la Red de Preincubadoras de la UCM.

- Realizar un proyecto piloto con el alumnado de la Facultad de EducaciónCFP (UCM) que quiera participar voluntariamente, de manera prioritaria con el estudiantado de cuarto curso de los grados en Pedagogía y Educación Social, vinculándolo al prácticum del último curso.

- Identificar y formar a docentes de la Facultad que quieran participar en el proyecto para que se conviertan en mentores.

- Contactar con Compluemprende UCM y con el Servicio de Orientación Universitaria de la Facultad de Educación-CFP (UCM) para desarrollar actividades conjuntas dentro del APE-Educación UCM. 


\subsection{Participantes}

Las personas usuarias del aula profesional de emprendimiento a las que iba destinada prioritariamente el APE-Educación UCM el curso 2020/2021 eran estudiantes de cuarto curso de los grados en Pedagogía y Educación Social, vinculando su desarrollo e implementación al prácticum de ambas titulaciones, con el objetivo de completar la formación práctica del alumnado con la información acerca de sus posibilidades para el emprendimiento. Estos perfiles fueron seleccionados por dos razones: primero porque en su actividad profesional encaja muy bien un planteamiento de emprendimiento, fundamentalmente de emprendimiento social; y, en segundo lugar, porque son profesiones muy versátiles que se desarrollan en nichos de mercado bastante innovadores. Sin embargo, la iniciativa despertó tanto interés que se unieron a esta estudiantes de otros grados, así como de máster y doctorado, ampliándose así el perfil de participantes. En el siguiente cuadro se especifica dicho perfil y sus intereses en materia de emprendimiento.

TABLA 4

Perfil del alumnado

\begin{tabular}{|l|l|l|}
\hline \multicolumn{1}{|c|}{ Género } & \multicolumn{1}{|c|}{ Estudios } & \multicolumn{1}{c|}{ Interés emprendedor } \\
\hline Femenino & Grado en Educación Social & $\begin{array}{l}\text { Proyecto: Actividades socioeducativas con per- } \\
\text { sonas autistas y con sus familias. }\end{array}$ \\
\hline Femenino & Grado en Educación Social & Iniciación al emprendimiento. \\
\hline Femenino & Grado en Educación Social & $\begin{array}{l}\text { Proyecto: Rehabilitación de la zona de Cam- } \\
\text { pamento en Madrid para uso y disfrute de la } \\
\text { ciudadanía. }\end{array}$ \\
\hline Femenino & Grado en Pedagogía & Proyecto: Educación y redes sociales. \\
\hline Femenino & Grado en Pedagogía & Proyecto: Educación y tercera edad. \\
\hline Femenino & Grado en Pedagogía & $\begin{array}{l}\text { Proyecto: Actividades formativas relacionadas } \\
\text { con educación infantil. }\end{array}$ \\
\hline Femenino & Grado en Pedagogía & Iniciación al emprendimiento. \\
\hline Femenino & $\begin{array}{l}\text { Grado en Pedagogía y Máster en Psi- } \\
\text { copedagogía }\end{array}$ & Proyecto: Gabinete psicopedagógico. \\
\hline
\end{tabular}




\begin{tabular}{|l|l|l|}
\hline \multicolumn{1}{|c|}{ Género } & \multicolumn{1}{|c|}{ Estudios } & \multicolumn{1}{|c|}{ Interés emprendedor } \\
\hline Femenino & $\begin{array}{l}\text { Grado en Maestro/a en Educación Pri- } \\
\text { maria }\end{array}$ & Iniciación al emprendimiento. \\
\hline Femenino & $\begin{array}{l}\text { Doble Grado en Maestro/a en Educa- } \\
\text { ción Infantil y Educación Primaria }\end{array}$ & Proyecto: Educación y nutrición. \\
\hline Femenino & $\begin{array}{l}\text { Doble Grado en Maestro/a en Educa- } \\
\text { ción Primaria y Pedagogía }\end{array}$ & Iniciación al emprendimiento. \\
\hline Femenino & $\begin{array}{l}\text { Máster Universitario en Arteterapia y } \\
\text { Educación Artística para la Inclusión } \\
\text { Social }\end{array}$ & Proyecto: Educación artística. \\
\hline Femenino & $\begin{array}{l}\text { Máster Universitario en Arteterapia y } \\
\text { Educación Artística para la Inclusión } \\
\text { Social }\end{array}$ & $\begin{array}{l}\text { Proyecto: Educación emocional a través del } \\
\text { Arte. }\end{array}$ \\
\hline Masculino & $\begin{array}{l}\text { Máster Universitario en Formación del } \\
\text { Profesorado de ESO y Bachillerato, FP } \\
\text { y Enseñanzas de Idiomas }\end{array}$ & $\begin{array}{l}\text { Proyecto: Emprendimiento derivado de la beca } \\
\text { Santander Explorer 2021. }\end{array}$ \\
\hline Femenino & Doctorado & Proyecto: Nutrición, educación y ciudadanía. \\
\hline
\end{tabular}

Fuente: elaboración propia

En la tabla 4 aparece el alumnado que mostró interés en participar en el proyecto y que cumplimentó un google form que se colgó en la página web del APE-Educación UCM. Son perfiles que pertenecen a diferentes grados y posgrados y que, además, muestran un interés por el emprendimiento también diferente, en algunos casos más encaminado a desarrollar proyectos concretos y en otros a iniciarse en temas de emprendimiento.

A este colectivo de participantes se unió más tarde el de mentores, que accedió a la información por la misma vía que el alumnado y que cumplimentó su correspondiente google form. Su perfil y experiencia en emprendimiento se precisan en la siguiente tabla. 
TABLA 5

Perfil y experiencia en emprendimiento de los docentes mentores

\begin{tabular}{|c|l|l|}
\hline Género & \multicolumn{1}{|c|}{ Perfil académico y profesional } & \multicolumn{1}{c|}{ Experiencia en emprendimiento } \\
\hline Femenino & $\begin{array}{l}\text { Máster en Psicoanálisis y Filosofía de } \\
\text { la Cultura. Profesora Asociada, espe- } \\
\text { cialista en Orientación Educativa. }\end{array}$ & $\begin{array}{l}\text { Interés en potenciar proyectos colaborativos } \\
\text { entre jóvenes para gestionar con autonomía su } \\
\text { futuro profesional. }\end{array}$ \\
\hline Femenino & $\begin{array}{l}\text { Máster en Filología Hispánica y Ense- } \\
\text { ñanza de Lengua Extranjera. } \\
\text { Profesora asociada, especialista en Di- } \\
\text { dáctica de la Lengua Inglesa. }\end{array}$ & $\begin{array}{l}\text { Participación en proyectos de innovación do- } \\
\text { cente en torno a la preparación de estudiantes } \\
\text { para las prácticas en el extranjero con un enfo- } \\
\text { que de emprendimiento. }\end{array}$ \\
\hline Femenino & $\begin{array}{l}\text { Máster Universitario en Dirección, } \\
\text { Gestión y Evaluación de Centros } \\
\text { Educativos y Máster en Humanidades. } \\
\text { Profesora asociada, especialista en Mé- } \\
\text { todos de Investigación en Educación. }\end{array}$ & $\begin{array}{l}\text { Especialista en gestión de calidad, dirección de } \\
\text { centros educativos, liderazgo y orientación aca- } \\
\text { démica, profesional y personal. Experiencia en } \\
\text { Pedagogías ágiles y formación en competencia } \\
\text { emprendedora. }\end{array}$ \\
\hline Masculino & $\begin{array}{l}\text { Doctor en Historia Moderna. Profesor } \\
\text { contratado doctor. Especialista en Di- } \\
\text { dáctica de las Ciencias Sociales. }\end{array}$ & $\begin{array}{l}\text { Gestión de proyectos, recepción de premios, } \\
\text { labores de asesoría, jornadas, tutoría de prác- } \\
\text { ticas y publicaciones sobre emprendimiento. }\end{array}$ \\
\hline Masculino & $\begin{array}{l}\text { Doctor en Pedagogía. Profesor contra- } \\
\text { tado doctor, especialista en Educación } \\
\text { Social y Pedagogía. }\end{array}$ & $\begin{array}{l}\text { Dirección de proyectos de innovación e inves- } \\
\text { tigación, gestión universitaria y experiencia en } \\
\text { aprendizaje-servicio. }\end{array}$ \\
\hline
\end{tabular}

Fuente: elaboración propia

La tabla 5 completa la información de las personas implicadas en este proyecto, incluyendo datos definitorios de los cinco docentes de la Facultad de Educación-CFP (UCM) que, tras la convocatoria pública, mostraron interés en participar en el APE-Educación UCM.

\subsection{Metodología y plan de trabajo}

La metodología cualitativa mediatiza el plan de trabajo del presente proyecto, el cual se desarrolla en tres fases, adaptadas de las establecidas en la obra de Bisquerra (2009) y consideradas fundamentales en un diseño de investigación de estas características. 
La primera fase se considera exploratoria y de reflexión. Esta fase se ha desarrollado durante los meses de septiembre y octubre de 2020 en la Facultad de Educación-CFP(UCM). En esta se llevó a cabo una sesión informativa con todo el equipo del proyecto y se desarrolló un seminario formativo de una mañana de duración, impartido el 8 de octubre de 2020 por la responsable del proyecto y una de las docentes implicadas en este, expertas ambas en emprendimiento, sobre las aulas profesionales de emprendimiento (qué son, qué características tienen, para qué sirven y cómo introducirlas en la Facultad de Educación-CFP de la UCM), los recursos físicos existentes (una hiperaula) y los informáticos necesarios (aula virtual y página web) para su implementación.

A continuación, se desarrolló la fase de planificación e implementación (trabajo de campo). Esta fase se llevó a cabo de noviembre de 2020 a abril de 2021 y se estructuró en tres partes.

En primer lugar, se diseñó un aula virtual de emprendimiento, la cual se creó a través de Moodle, así como la página web APE-Educación UCM y su logotipo, poniéndose a disposición de la Facultad de Educación-CFP (UCM) para su vinculación a la página web institucional de esta como un espacio de coworking, útil para reuniones de trabajo con mentores, encuentros con inversores o pitchs de proyectos. Esta aula se pretendía combinar con una física y disponible en la Facultad de Educación-CFP (UCM) para ser usada en el momento en el que la situación sanitaria lo permitiera. Nos referimos a la hiperaula, un espacio único con posibilidades múltiples para la agrupación del estudiantado y tecnología puntera, un marco incomparable para el trabajo individual o en grupo, donde el alumnado emprendedor puede trabajar en su proyecto, con conexión a internet por red wifi, pizarras blancas para cada proyecto, ordenadores de uso común para realizar consultas y mobiliario flexible.

En segundo lugar, se organizó un webinar, el 15 de diciembre de 2020, con el objetivo de dar a conocer el proyecto a toda la comunidad universitaria de la Facultad de Educación-CFP (UCM), así como presentar su página web y el APE-Educación UCM virtual. Contó con la participación del equipo decanal y el Servicio de Orientación Universitaria de la Facultad, con Compluemprende UCM, la Asociación pro Colegio de Pedagogía y Psicopedagogía de la Comunidad de Madrid (PROCOLPED) y el equipo completo del proyecto. Este conjunto de participantes permitió visibilizar la iniciativa, darla a conocer a sus destinatarios, así como compartir con la comunidad universitaria 
todas sus posibilidades profesionales y apoyos institucionales. Se utilizó para ello el entorno virtual del que dispone el campus de la UCM y Google Meet, herramientas muy útiles en tiempos de pandemia. Y es en este momento en el que se presentan los google form para estudiantes y docentes (a ellos nos hemos referido con anterioridad), en los que se solicita su participación en el proyecto. Se apuntan 15 estudiantes y 5 mentores, asignándose inicialmente tres estudiantes por mentor, en función de los intereses manifestados por el alumnado participante y el currículo y experiencia en emprendimiento de los docentes mentores (la información al respecto se encuentra en las tablas 4 y 5). Todo ello se visibiliza en el APE-Educación UCM virtual.

En tercer lugar, se desarrollaron dos actividades formativo-informativas que se pueden consultar en la página web del proyecto y en el APE-Educación UCM virtual:

- Diseño e implementación de una píldora formativa impartida por los dos miembros del equipo del proyecto que se dedican profesionalmente a temas de emprendimiento y destinada tanto a estudiantes como a docentes de la Facultad de Educación-CFP (UCM). Esta píldora formativa se llevó a cabo el 5 de marzo de 2021 a través de un webinar que tenía por título "Por qué no tienes que buscar empleo". Su objetivo era motivar al emprendimiento y dar pautas para llevarlo a cabo con éxito.

- El 16 de abril de 2021, por su parte, se organizaron dos sesiones informativas, una por la mañana para el estudiantado participante en el proyecto y otra por la tarde para sus mentores, con el objetivo de aclarar las dudas existentes con relación al papel de cada perfil en el proyecto. En dichas sesiones, el alumnado informó acerca de sus propuestas e intereses en el ámbito del emprendimiento y los docentes mentores dieron un feedback de su labor como asesores del estudiantado implicado, indicando los procesos de seguimiento y acompañamiento llevados a cabo para el análisis de la idea de negocio, el establecimiento de hitos y la recomendación de actuaciones.

Y la última fase ha consistido en la evaluación final del proyecto y el análisis de resultados. A esta fase nos vamos a referir a continuación de manera más detallada. 


\subsection{Evaluación y análisis de resultados}

Esta fase se ha desarrollado entre mayo y junio de 2021. En ella se ha llevado a cabo la recogida y el análisis de la información, identificando el número de estudiantes que se han apuntado y los resultados obtenidos en cuanto a formación y propuestas de negocio, el número de mentores y su implicación como guías del alumnado, así como el éxito del aula virtual de emprendimiento.

Como resultados concretos conseguidos podemos enumerar los siguientes:

- Número de estudiantes que han participado: se apuntaron 15 estudiantes, 14 mujeres y un hombre. 11 tenían un proyecto ya pensado y 4 interés por aprender sobre el emprendimiento. En cuanto a sus estudios, 10 cursaban estudios de grado (dos un doble grado) y 5 de posgrado (una persona ha cursado un grado y ahora está cursando un máster). De los 15 estudiantes, 3 ( 2 sin una idea de emprendimiento clara y una con interés en posponer el desarrollo de su proyecto) se dieron de baja a finales de abril, permaneciendo en el proyecto 12 (de los cuales 2 no tenían aún una idea de negocio). Al estudiantado se le ofreció formación sobre la herramienta Lean Canvas (el instrumento para comenzar a hacer viable una idea y transformarla en un proyecto de emprendimiento) y se le informó sobre la oferta formativa de Compluemprende UCM y del Servicio de Orientación Universitaria de la Facultad de Educación-CFP (UCM). Entre los proyectos en marcha merecen ser destacados los siguientes: educación y redes sociales y rehabilitación de la zona de Campamento en Madrid para uso y disfrute de la ciudadanía.

- Número de mentores: se apuntaron cinco mentores inicialmente, pero desde finales de abril se han mantenido solo cuatro, que han realizado tareas de asesoramiento a estudiantes. Cada mentor ha atendido a 3 estudiantes (ya que se ha reducido el número de participantes a 12); siendo el alumnado quien ha elegido a sus mentores una vez consultado su currículo en el aula virtual APE-Educación UCM.

- Aula virtual de emprendimiento: el aula se ha convertido en un punto de referencia para estudiantes y mentores, pues recoge toda la información sobre los proyectos del alumnado, el currículo del profesorado, los eventos formativos e informativos llevados a cabo, así como todas las características del proyecto y sus fases. Dicha aula ha servido, además, 
como vía de comunicación, la cual se ha completado con la utilización del correo electrónico para hacer llegar todas las novedades a estudiantes, mentores y el resto de la comunidad universitaria de la Facultad de Educación-CFP (UCM).

Finalmente, los resultados del proyecto se presentan el 17 de junio de 2021, en sesión abierta y virtual, a todo el equipo, al alumnado participante y al profesorado implicado como mentores, así como al Servicio de Orientación Universitaria de la Facultad de Educación-CFP (UCM), a Compluemprende UCM y a toda la comunidad universitaria de la Facultad de Educación-CFP (UCM). Se organiza para ello un webinar que cuenta con la participación del señor decano de la Facultad de Educación-CFP (UCM) y de un representante del Vicerrectorado de Empleabilidad y Emprendimiento de la UCM, institucionalmente, así como con la participación de tres mentores y una alumna emprendedora que informan sobre el desarrollo del proyecto, los logros conseguidos y las perspectivas de futuro. Dicho webinar se cuelga en la página web del APE-Educación UCM para facilitar su consulta. Asimismo, gracias a la invitación recibida de Compluemprende UCM, se realiza una presentación de los logros del proyecto en el panel "Special Session: Entrepreneurship in Humanities \& Education", el día 2 de julio de 2021, en la Facultad de Educación-CFP (UCM) y en el marco del Nineteenth International Conference on New Directions in the Humanities.

Todo ello nos permite concluir que se han conseguido los objetivos planteados al inicio del proyecto, tanto los generales como los específicos:

- Se ha realizado un trabajo interdisciplinar, aunando educación y empresa.

- Se ha logrado sensibilizar a la comunidad universitaria de la Facultad de Educación-CFP (UCM) sobre el tema del emprendimiento, lo que se ha traducido en el interés mostrado por un conjunto de estudiantes y docentes en participar en el proyecto.

- Se ha creado el aula virtual de emprendimiento APE-Educación UCM y esperamos contar el curso próximo con el aula física (hiperaula).

- Se ha conformado un grupo de participantes de los grados considerados como prioritarios y además se ha completado dicho grupo con otros perfiles. 
- Se han puesto en marcha los pilares para conseguir una red de mentores.

- Se ha contado con la colaboración de Compluemprende UCM y el Servicio de Orientación Universitaria de la Facultad de Educación-CFP (UCM), que han sido un gran apoyo en la divulgación y la formación del alumnado participante.

En último término, la buena recepción del APE-Educación UCM ha animado al equipo a solicitar un nuevo proyecto Innova Docencia para el curso 2021/2022, que le ha sido concedido, el cual pretende consolidar el APE virtual de la Facultad de Educación-CFP (UCM) como un servicio permanente y sostenible, abierto a estudiantes y, ahora también, a alumni, integrado en el ecosistema emprendedor UCM, que se pueda combinar con un aula física y disponible en la Facultad de Educación-CFP(UCM): la hiperaula. En este sentido, en esta nueva edición queremos incluir una visión sostenible del emprendimiento, siguiendo la estrategia española de desarrollo sostenible establecida en la Agenda 2030, de tal forma que las ideas de negocio que se generen en la preincubadora (APE-Educación UCM) reporten un beneficio económico, social y medioambiental que satisfaga las necesidades del presente sin comprometer las necesidades del futuro.

\section{Conclusiones}

La idea de impulsar la economía desde el emprendimiento, la importancia de vincular el conocimiento con la empresa y la necesidad de promover la creación de empleo entre la juventud, unido al antecedente positivo de las aulas profesionales de emprendimiento (APE) en la Formación Profesional, han llevado a la Facultad de Educación-CFP de la UCM a plantearse el desarrollo de su propia APE.

Esta idea surge de un enfoque educativo del emprendimiento que pretende promover el desarrollo del potencial emprendedor del alumnado, apoyado en su iniciativa, autonomía, responsabilidad y madurez personal, para que valore, piense, invente, cree e imagine nuevas fórmulas y proyectos personales para afrontar los amplios desafíos que se nos plantean, específicamente en el mundo 
del trabajo. Este enfoque se entronca en los planteamientos europeos sobre emprendimiento herederos de las ideas de crecimiento, competitividad y empleo de Delors (1993), y se comienza a vislumbrar en la Universidad española a través de la aparición de diferentes infraestructuras de emprendimiento en facultades y centros de educación superior.

Dentro de este engranaje surge el APE-Educación UCM, una propuesta de emprendimiento novedosa, la primera en una Facultad de Educación de la Comunidad Autónoma de Madrid, que busca aproximar el emprendimiento a un entorno profesional donde aún no tiene demasiados seguidores y que pretende consolidarse como un servicio de apoyo de la comunidad universitaria a la inserción laboral de su alumnado.

Su origen debemos situarlo en un Proyecto Innova Docencia de la UCM, una vía que se abre al profesorado universitario para poner en marcha propuestas novedosas para el estudiantado y la institución. Se apoya en un equipo interdisciplinar implicado y preocupado por temas de emprendimiento. Un equipo que integra perfiles docentes, discentes y profesionales con el objetivo de no dejar nada al azar. Se ha desarrollado durante el curso 2020/2021, pero pretende consolidarse en la Facultad de Educación-CFP y llegar a formar parte de la red de preincubadoras de la UCM. Ha sido recibido con bastante interés, pues se han apuntado 15 estudiantes y 5 docentes a una iniciativa particular y de escasa tradición en los estudios universitarios de educación, manteniendo la continuidad de la mayor parte de ambos perfiles, en un curso como este, especialmente duro y complejo debido a la pandemia. Además, ha sido bien recibida por la comunidad universitaria, ya que tanto Compluemprende-UCM como el Servicio de Orientación Universitaria de la Facultad de EducaciónCFP, su equipo decanal y docentes y estudiantes de diferentes perfiles, han acudido a los webinar formativo-informativos y han mostrado interés en temas de emprendimiento; apostando por su continuidad gracias a la solicitud y concesión de un nuevo Proyecto Innova Docencia para el curso 2021/2022.

En definitiva, queremos mostrar nuestra satisfacción por la acogida de una propuesta necesaria pero desconocida, útil pero con escasa tradición, y apoyada a nivel autonómico, nacional y europeo, pero habitualmente de manera más teórica que práctica. 


\section{Agradecimientos}

Esta publicación está enmarcada dentro del Proyecto "Formación del Potencial Emprendedor. Generación de un Modelo Educativo de Identidad Emprendedora", con Referencia PID2019-104408GB-I00, dentro del Plan Estatal 2017-2020 de Proyectos I+D+i (Generación de Conocimiento), financiado por el Ministerio de Ciencia e Innovación - Agencia Estatal de Investigación/10.13039/501100011033.

\section{BiBLIOGRAFÍA}

Azqueta, A. (2021). La educación emprendedora y las instituciones internacionales: respuestas a un desafío global, en A. Bernal (ed.), Educación emprendedora (pp. 35-60). Madrid: Síntesis.

AzQueta, A. y Naval, C. (2019). Educación para el emprendimiento: una propuesta para el desarrollo humano. Revista Española de Pedagogía 77(274), 517-533. DOI: https://doi.org/10.22550/REP77-3-2019-03

Bisquerra, R. (coord.) (2009). Metodología de investigación educativa. Madrid: La Muralla.

Bourgeois, A. (2011). Entrepreneurship Education at School in Europe: National Strategies, Curricula and Learning Outcomes. Education, Audiovisual and Culture Executive Agency. Bruselas: European Commission.

Comisión de las Comunidades Europeas (2002). Libro verde. El espíritu empresarial en Europa. Recuperado de: https://bit.ly/36i6XFi

Comisión de las Comunidades Europeas (2004). Plan de acción. El programa europeo en favor del espiritu empresarial. Recuperado de: https:// bit.ly/2UtCEZH

Comisión Europea (2000). Carta Europea de la Pequeña Empresa. Recuperado de: https://bit.ly/3hCRHYR

COMISIÓN EUROPEA (2013). Entrepreneurship 2020 action plan: reigniting the entrepreneurial spirit in Europe. Recuperado de: https://bit.ly/36dn7jg

Comité Europeo de las Regiones (2009). The European Entrepreneurial Region. Recuperado de: https://bit.ly/3xhLWq2 
Compluemprende (2017). Informe de Actividad 2016-2017. Recuperado de: https://n9.cl/30m7

Compluemprende (2019). Informe de Actividad 2018-2019. Recuperado de: https://n9.cl/oex2c

DeCRETo 63/2019, de 16 de julio, del Consejo de Gobierno, por el que se regula la ordenación y organización de la formación profesional en la Comunidad de Madrid. BOCM 170, 10-36.

Decreto Rectoral 1/2021, de 11 de enero, de establecimiento de los Vicerrectorados de la Universidad Complutense de Madrid, de delegación de competencias y de diversas cuestiones de índole organizativo. BOCM 11, 91- 103.

Decreto Rectoral 1/2007, de 2 de enero, por el que se crea la oficina complutense del emprendedor. BOUC 1, 1-4.

Delors, J. (1993). Crecimiento, competitividad y empleo. Retos y pistas para entrar en el siglo XXI. Libro Blanco. Luxemburgo: Oficina de Publicaciones Oficiales de las Comunidades Europeas.

DOCE (2006). Programa marco para la innovación y la competitividad. Recuperado de: https://bit.ly/3ysAI23

Gobierno de España (2021). Plan de Recuperación, Transformación y Resiliencia. Recuperado de: https://n9.cl/7p8qa

Mayor-Zaragoza, F. (2009) Los límites del crecimiento. Temas para el Debate 181, 10-16.

Morales, M., Mira, G. y Arias, M. (2010). Enfoques y retos de la función de extensión universitaria como mecanismos de integración: Universidad, Empresa, Estado. II Congreso Internacional de Gestión Tecnológica e Innovación. 7 y 8 de octubre de 2010. Bogotá D.C., Colombia.

ORDEN 1378/2021, de 19 de mayo, de la Consejería de Educación y Juventud, por la que se regulan las aulas profesionales de emprendimiento en los centros educativos que impartan enseñanzas de formación profesional en la Comunidad de Madrid. BOCM 129, 10-14.

REDIE (2015). La educación para el emprendimiento en el sistema educativo. Principado de Asturias: Secretaría Técnica del Ministerio de Educación, Cultura y Deporte.

Sanabria, P. E., Morales, M. E. y Ortiz, C. (2015). Interacción universidad y entorno: marco para el emprendimiento. Educ. Educ. 18(1), 111-134. 
Suárez-Ortega, M., SÁnchez-García, M. F. y Soto-González, M.D. (2020). Desarrollo de la carrera emprendedora: Identificación de perfiles, competencias y necesidades. Revista Complutense de Educación 31(2), 173-184. Recuperado de: https://dx.doi.org/10.5209/rced.62001

Universidad Complutense de Madrid (2020). Convocatoria Proyectos de Innovación. Grupos docentes, centros y servicios. Madrid: Vicerrectorado de Calidad (UCM).

VÁzquez, C. (2017). Educación para el emprendimiento en la universidad. Estudios de la Gestión: Revista Internacional de Administración 2, 121-147. 